\title{
The Glue Content of the Pion
}

\author{
Harvey B. Meyer*, John W. Negele \\ Center for Theoretical Physics \\ Massachusetts Institute of Technology \\ Cambridge, MA 02139 U.S.A. \\ E-mail: meyerh@mit.edu, negele@mit.edu
}

\begin{abstract}
We perform a quenched computation of the glue momentum fraction in the pion. Different discretizations of the gluonic energy-momentum tensor are studied on the lattice for that purpose. We discuss some implications based on the momentum sum rule. Finally we point out promising applications of the techniques developed here.
\end{abstract}

MIT-CTP 3872

The XXV International Symposium on Lattice Field Theory

July 30-4 August 2007

Regensburg, Germany

*Speaker. 


\section{Introduction}

A striking feature of QCD is the large contribution of gluons to the mass and momentum of hadrons, so it is of fundamental interest to calculate the contributions of gluons from first principles using lattice QCD. The first moments

$$
\begin{aligned}
\langle x\rangle_{\mathrm{f}}\left(q^{2}\right) & \equiv \sum_{f=u, d, s} \int_{0}^{1} x d x\left\{\bar{f}\left(x, q^{2}\right)+f\left(x, q^{2}\right)\right\} \\
\langle x\rangle_{\mathrm{g}}\left(q^{2}\right) & \equiv \int_{0}^{1} x d x g\left(x, q^{2}\right)
\end{aligned}
$$

of the quark and gluon distribution functions $f(x), \bar{f}(x)(f=u, d, s, \ldots)$ and $g(x)$ acquire a precise field-theoretic meaning via the operator product expansion in QCD. They satisfy the well-known momentum sum rule (MSR) $\langle x\rangle_{\mathrm{f}}\left(q^{2}\right)+\langle x\rangle_{\mathrm{g}}\left(q^{2}\right)=1$ and are related to the corresponding contributions to the energy-momentum tensor $T_{\mu \nu}$ evaluated on the hadronic state. Separating the traceless part $\bar{T}_{\mu \nu}$ from the trace part $S$ for gluons, denoted ' $\mathrm{g}$ ', and quarks, denoted 'f', $T_{\mu \nu}$ has the explicit form

$$
\begin{aligned}
T_{\mu v} & \equiv \bar{T}_{\mu v}^{\mathrm{g}}+\bar{T}_{\mu v}^{\mathrm{f}}+\frac{1}{4} \delta_{\mu v}\left(S^{\mathrm{g}}+S^{\mathrm{f}}\right), \\
\bar{T}_{\mu v}^{\mathrm{g}} & =\frac{1}{4} \delta_{\mu v} F_{\rho \sigma}^{a} F_{\rho \sigma}^{a}-F_{\mu \alpha}^{a} F_{v \alpha}^{a}, \\
\bar{T}_{\mu v}^{\mathrm{f}} & =\frac{1}{4} \sum_{f} \bar{\psi}_{f} \stackrel{\leftrightarrow}{D_{\mu}} \gamma_{v} \psi_{f}+\bar{\psi}_{f} \stackrel{\leftrightarrow}{D_{v}} \gamma_{\mu} \psi_{f}-\frac{1}{2} \delta_{\mu v} \bar{\psi}_{f} \stackrel{\leftrightarrow}{D_{\rho}} \gamma_{\rho} \psi_{f}, \\
S^{\mathrm{g}} & =\beta(g) /(2 g) F_{\rho \sigma}^{a} F_{\rho \sigma}^{a}, \quad S^{\mathrm{f}}=\left[1+\gamma_{m}(g)\right] \sum_{f} \bar{\psi}_{f} m \psi_{f}
\end{aligned}
$$

where $\stackrel{\leftrightarrow}{D_{\mu}}=\overrightarrow{D_{\mu}}-\overleftrightarrow{D_{\mu}}, \beta(g)=-b_{0} g^{3}+\ldots$ is the beta-function with $b_{0}=\left(\frac{11}{3} N-\frac{2}{3} N_{\mathrm{f}}\right)(4 \pi)^{-2}, \gamma_{m}(g)$ is the anomalous dimension of the mass operator, and all expressions are written in Euclidean space. The gauge action in this notation is $\frac{1}{4} F_{\mu \nu}^{a} F_{\mu \nu}^{a}$. For an on-shell particle with four-momentum $p=\left(i E_{p}, \mathbf{p}\right), E_{\mathbf{p}}^{2}=M^{2}+\mathbf{p}^{2}$, we have the relations

$$
\begin{aligned}
\left\langle\Psi, \mathbf{p}\left|\int d^{3} \mathbf{z} \bar{T}_{00}^{\mathrm{f}, \mathrm{g}}(z)\right| \Psi, \mathbf{p}\right\rangle & =\left[E_{\mathbf{p}}-\frac{1}{4} M^{2} / E_{\mathbf{p}}\right]\langle x\rangle_{\mathrm{f}, \mathrm{g}}, \\
\left\langle\Psi, \mathbf{p}\left|\int d^{3} \mathbf{z} S^{\mathrm{f}, \mathrm{g}}(z)\right| \Psi, \mathbf{p}\right\rangle & =\left(M^{2} / E_{\mathbf{p}}\right) b_{\mathrm{f}, \mathrm{g}}, \\
\langle x\rangle_{\mathrm{f}}+\langle x\rangle_{\mathrm{g}} & =b_{\mathrm{f}}+b_{\mathrm{g}}=1,
\end{aligned}
$$

where states are normalized according to $\langle\mathbf{p} \mid \mathbf{p}\rangle=1$. The renormalization of $\langle x\rangle_{\mathrm{f}, \mathrm{g}}$ will be discussed in section 4 .

Equation 1.7 shows that in the infinite momentum frame, where $E_{p} \sim P \rightarrow \infty,\langle x\rangle_{g}$ represents the momentum fraction arising from gluons, and calculating $\langle x\rangle_{g}$ is the main goal of this work. In the rest frame, the gluon contribution of Eq. 1.7 to the hadron mass is $\frac{3}{4} M\langle x\rangle_{g}$ [1]. From Eq. 1.8 in the rest frame, the contribution of the trace anomaly $S^{g}$ to the hadron mass is $\frac{1}{4} b_{g} M$ [1], and we have performed the first step to calculate this matrix element as well [3]. Finally, we remark that in thermodynamics at temperature $T$ the energy density $\varepsilon$ and pressure $P$ are given by

$$
\varepsilon-3 P=\langle S\rangle_{T}-\langle S\rangle_{0}, \quad \varepsilon+P=\frac{4}{3}\left\langle\bar{T}_{00}\right\rangle_{T} .
$$

The pioneering calculation of the glue momentum fraction (in the proton) was presented at the LATTICE96 conference [2]. In the present study we treat the case of "heavy pions" with masses in the range $600 \mathrm{MeV}<M_{\pi}<1060 \mathrm{MeV}$. Our improved techniques, applied here in the quenched approximation, are applicable in full QCD calculations, and to the case of the proton. 


\section{Discretization and normalization of the gluonic operators}

We use the Wilson gluon action $\frac{1}{g_{0}^{2}} \sum_{x, \mu \neq \nu} \operatorname{Tr}\left\{1-P_{\mu \nu}(x)\right\}$ and the Wilson fermion action [4]. There are two distinct ways [6] to discretize the Euclidean gluonic operator $\bar{T}_{00}^{\mathrm{g}}=\frac{1}{2}\left(-\mathbf{E}^{a} \cdot \mathbf{E}^{a}+\right.$ $\left.\mathbf{B}^{a} \cdot \mathbf{B}^{a}\right)$ and the trace anomaly $S^{\mathrm{g}}=\frac{\beta(g)}{g}\left(\mathbf{E}^{a} \cdot \mathbf{E}^{a}+\mathbf{B}^{a} \cdot \mathbf{B}^{a}\right)$ on a hypercubic lattice.

The first, denoted 'bp' for bare-plaquette, uses a sum of bare plaquettes $P_{\mu v}$ around a bodycentered point $x_{\odot}=x+\frac{1}{2} a \sum_{\mu} \hat{\mu}$ :

$$
\begin{aligned}
& \bar{T}_{00}^{\mathrm{bp}}\left(x_{\odot}\right) \equiv \chi^{\mathrm{bp}}\left(g_{0}\right) \frac{Z_{\mathrm{g}}\left(g_{0}\right)}{8 a^{4} g_{0}^{2}} \sum_{\mathscr{P}} s_{00}(0 \mathscr{P}, 1 \mathscr{P}) \sum_{\omega, \lambda=0,1} \operatorname{Tr}\left\{1-P_{0_{\mathscr{P}} 1_{\mathscr{P}}}(x+a \lambda \widehat{2 \mathscr{P}}+a \omega \widehat{3 \mathscr{P}})\right\} \\
& S^{\mathrm{bp}}\left(x_{\odot}\right) \equiv \chi_{s}^{\mathrm{bp}}\left(g_{0}\right) \frac{d g_{0}^{-2}}{d \log a} \frac{1}{8 a^{4}} \sum_{\mathscr{P}} \sum_{\omega, \lambda=0,1} \operatorname{Tr}\left\{1-P_{0_{\mathscr{P}} 1_{\mathscr{P}}}(x+a \lambda \widehat{2 \mathscr{P}}+a \omega \widehat{3 \mathscr{P}})\right\}
\end{aligned}
$$

where $\mu_{\mathscr{P}}$ is the image under permutation $\mathscr{P}$ of $\mu$ and $s_{00}(\mu, v)$ is 1 if $(\mu, v)$ are both spatial and -1 otherwise. Other diagonal elements of the energy-momentum tensor are obtained by letting the other directions play the role of time. When summed over a time-slice, these expressions simplify to Eq. 7 of Ref. [3]. The other form ('bare clover') is

$$
\begin{gathered}
\bar{T}_{00}^{\mathrm{bc}}(x) \equiv \frac{\chi^{\mathrm{bc}}\left(g_{0}\right) Z_{\mathrm{g}}\left(g_{0}\right)}{g_{0}^{2}} \operatorname{Re} \operatorname{Tr}\left[\sum_{k}\left(\widehat{F}_{0 k}\right)^{2}-\sum_{k<l}\left(\widehat{F}_{k l}\right)^{2}\right] \\
S^{\mathrm{bc}}(x) \equiv \chi_{s}^{\mathrm{bc}}\left(g_{0}\right) \frac{d g_{0}^{-2}}{d \log a} \operatorname{Re} \operatorname{Tr}\left[\sum_{k}\left(\widehat{F}_{0 k}\right)^{2}+\sum_{k<l}\left(\widehat{F}_{k l}\right)^{2}\right],
\end{gathered}
$$

where $\widehat{F}_{\mu v}(x)$ is the clover-shaped discretization of the field-strength tensor (see []). This form allows for the discretizations of off-diagonal elements of $\bar{T}_{\mu \nu}$ as well. Each of the normalization factors $Z_{\mathrm{g}}\left(g_{0}\right), \chi^{\mathrm{bc}}\left(g_{0}\right)$ and $\chi_{s}^{\mathrm{bc}}\left(g_{0}\right)$ in Eq. (2.1,2.3) is of the form $1+\mathrm{O}\left(g_{0}^{2}\right)$. The factor $Z_{\mathrm{g}}\left(g_{0}\right)$ is dictated by an exact lattice sum-rule for the Wilson gauge action and is known with a precision of about 1\% (see [10] and Refs. therein). To obtain the absolute normalization of other discretizations, it is sufficient to compute their normalization $\chi\left(g_{0}\right)$ relative to that of the bare plaquette.

New versions of the gluonic operators are obtained by replacing the link variables in Eq. (2.12.4) by 'smeared' versions thereof. The HYP form of smearing [9] is particularly attractive in the present context in that it preserves the symmetry among all four Euclidean directions.

Our criteria for the choice of the discretization are to maximize the signal-to-noise ratio, minimize cutoff effects, and preserve locality as much as possible. We studied the signal-to-noise ratio for four different discretizations by comparing the variance of the entropy at temperature $T=1 / L_{0}=1.21 T_{c}$ [7], which is proportional to the expectation value of $\sum_{x} \bar{T}_{00}(x)$, on $L_{0} \times L^{3}$ lattices of fixed physical size.

The resulting variances for the plaquette and clover discretizations with bare and HYP links are shown in Table 1. We find dramatic differences between the discretizations, with the HYPclover operator reducing the variance by almost two orders of magnitude as compared to the bare plaquette operator. Variance reduction comes at the cost of a certain loss of locality, since the HYP plaquette and HYP-clover operators have extent $3 a$ and $4 a$ respectively. As Fig. (1, left panel) illustrates, the variance of $\sum_{x} \bar{T}_{00}^{\mathrm{g}}(x)$ grows like $a^{-4}$, but the prefactor is non-universal and depends 


\begin{tabular}{|c|c|cc|cc|}
\cline { 3 - 6 } \multicolumn{2}{c|}{} & \multicolumn{2}{c|}{ relative variance } & \multicolumn{2}{c|}{ normalization } \\
\cline { 3 - 6 } \multicolumn{2}{c|}{} & bare & HYP & bare & HYP \\
\hline$\beta=6.000$ & plaq. & $26.42(71)$ & $0.6518(43)$ & 1.000 & $0.5489(68)$ \\
$6 \times 16^{3}$ & clover & $3.85(11)$ & $0.3049(41)$ & $2.184(67)$ & $0.613(20)$ \\
\hline$\beta=6.093$ & plaq. & $45.5(2.5)$ & $0.991(11)$ & 1.000 & $0.546(14)$ \\
$7 \times 20^{3}$ & clover & $6.20(14)$ & $0.4393(40)$ & $2.026(52)$ & $0.585(16)$ \\
\hline$\beta=6.180$ & plaq. & $81.5(5.8)$ & $1.940(26)$ & 1.000 & $0.596(20)$ \\
$8 \times 22^{3}$ & clover & $11.84(33)$ & $0.8280(85)$ & $2.092(68)$ & $0.636(22)$ \\
\hline$\beta=6.295$ & plaq. & $157(16)$ & $3.080(50)$ & 1.000 & $0.563(28)$ \\
$9 \times 24^{3}$ & clover & $19.74(75)$ & $1.268(16)$ & $1.928(93)$ & $0.598(30)$ \\
\hline$\beta=6.408$ & plaq. & $346(81)$ & $7.67(29)$ & 1.000 & $0.617(68)$ \\
$11 \times 29^{3}$ & clover & $43.3(3.6)$ & $3.042(78)$ & $1.91(20)$ & $0.654(75)$ \\
\hline
\end{tabular}

Table 1: Left: the relative variance, $\left\langle\mathscr{O}^{2}\right\rangle /\langle\mathscr{O}\rangle^{2}-1$, of the operators $\mathscr{O}=\sum_{x} \bar{T}_{00}^{\mathrm{g}}(x)$ on lattices of matched physical sizes for different discretizations described in the text. Right: the normalization $\chi\left(g_{0}, a / L_{0}\right)$ of the operator relative to the bare plaquette, determined on the same lattice.
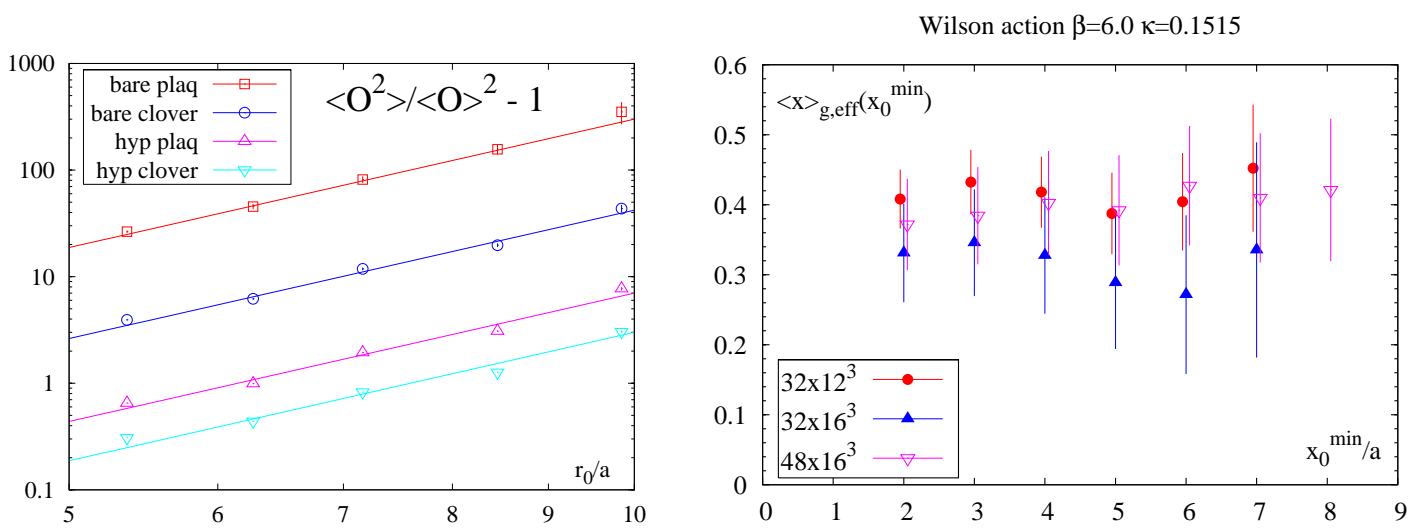

Figure 1: Left: the relative variance, $\left\langle\mathscr{O}^{2}\right\rangle /\langle\mathscr{O}\rangle^{2}-1$, of the operator $\mathscr{O}=\sum_{x} \bar{T}_{00}^{\mathrm{g}}(x)$ as listed in Table 1. The lines of the form $y=a x^{4}$ are drawn to guide the eye. Right: The effective gluonic momentum fraction $\langle x\rangle_{\mathrm{g} \text {,eff }}^{(\pi)}\left(x_{0}^{\mathrm{min}}\right)$ in a heavy pion, $M_{\pi} \simeq 1060 \mathrm{MeV}$, using the HYP-plaquette discretization.

strongly on the discretization. As a compromise between locality and variance reduction, from now on we work with the HYP-plaquette operator. In [3], we performed a check of its discretization errors by computing the dependence of $\chi$ on $a / L_{0}$ at $\beta=6$. We showed that the dependence of $\chi$ on $a / L_{0}$ is mild and statistically consistent with zero for $L_{0} / a \geq 6$.

The $\chi$ normalization factors are given in Tab. 1 for the four discretizations. We have thus determined the non-perturbative normalization of $\bar{T}_{00}^{\mathrm{g}}$ in a range of lattice spacings [⿰], $5<r_{0} / a<10$. As a check of the correct normalization of the chosen HYP-plaquette operator, we computed its expectation value on the lightest scalar glueball. In that case, we know that the momentum fraction carried by the glue is one, and indeed we find $\langle x\rangle_{\mathrm{g}}^{(G)}=1.16(18)$. 


\begin{tabular}{|c|cccc|}
\hline$M_{\pi}(\mathrm{Mev})$ & $32 \cdot 12^{3}$ & $32 \cdot 16^{3}$ & $48 \cdot 16^{3}$ & $24^{4}$ \\
\hline $1060(10)$ & $0.39(6)_{23091}$ & $0.29(9)_{7113}$ & $0.40(8)_{8331}$ & $0.34(9)_{1048}$ \\
$891(9)$ & - & - & - & $0.36(8)_{3066}$ \\
$624(6)$ & - & - & - & $0.58(16)_{2538}$ \\
\hline
\end{tabular}

Table 2: The glue momentum fraction $\langle x\rangle_{\mathrm{g}}^{(\pi)}$ in the pion. The integer in each subscript denotes the number of configurations used.

\section{The glue momentum fraction in the pion}

We consider a triplet of Wilson quarks, labeled $u, d, s$, with periodic boundary conditions in all directions and with common $\kappa=0.1515,0.1530$ and 0.1550 corresponding to pion masses approximately 1060,890 and $620 \mathrm{MeV}$ on the $\beta=6$ lattices $32 \cdot 12^{3}, 32 \cdot 16^{3}, 48 \cdot 16^{3}$ and $24^{4}$. We use $r_{0}=0.5 \mathrm{fm}$ to set the scale. To calculate the gluonic momentum fraction in the pion, we define the effective momentum fraction

$$
\langle x\rangle_{\mathrm{g}, \mathrm{eff}}^{(\pi)}\left(x_{0}^{\mathrm{min}}\right) \equiv \frac{8}{3 M_{\pi}} \frac{a^{3}}{\left|\Lambda_{0}\right|} \times \sum_{\mathbf{x} ; x_{0} \in \Lambda_{0}}\left[\frac{\sum_{\mathbf{y}}\left\langle j(0) \bar{T}_{00}^{\mathrm{hp}}\left(x_{\odot}\right) j\left(\frac{L_{0}}{2}, \mathbf{y}\right)\right\rangle}{\sum_{\mathbf{y}^{\prime}}\left\langle j(0) j\left(\frac{L_{0}}{2}, \mathbf{y}^{\prime}\right)\right\rangle}-\left\langle\bar{T}_{00}^{\mathrm{hp}}\left(x_{\odot}\right)\right\rangle\right],
$$

where $\Lambda_{0}=\left\{x_{0}^{\min }, \ldots, \frac{L_{0}}{2}-x_{0}^{\min }-a, \frac{L_{0}}{2}+x_{0}^{\min }, \ldots, L_{0}-x_{0}^{\min }-a\right\}$. For large $L_{0}$ and $x_{0}^{\min },\langle x\rangle_{\mathrm{g}, \mathrm{eff}}^{(\pi)} \rightarrow\langle x\rangle_{\mathrm{g}}^{(\pi)}$. As a source field for the pion, we use the isovector pseudoscalar density $j(x)=\bar{d}(x) \gamma_{5} u(x)$. Its twopoint function is positive on every configuration, for each of which we do 12 inversions corresponding to Dirac and color indices. On a $24^{4}$ lattice, we take advantage of the symmetry between all directions to perform these inversions at the points $k(6,6,6,6)$ for $k=0,1,2,3$ and symmetrize expression (3.1) with respect to all directions, so that $\sum_{x, \mu} \bar{T}_{\mu \mu}(x)$ vanishes on every configuration. The right panel of Fig. 1 1 shows our stable plateaus for $\langle x\rangle_{\mathrm{g}, \text { eff }}^{(\pi)}$ at large values of $x_{0}^{\min }$ for three lattice sizes, and the results are summarized in Tab. 2.

The lattice equivalent of Eq. 1.9 has been derived for Wilson lattice QCD in [11]. It reads

$$
1+\mathrm{O}\left(a^{2}\right)=\langle x\rangle_{\mathrm{g}}+\langle x\rangle_{\mathrm{f}}, \quad\langle x\rangle_{\mathrm{f}}=Z_{\mathrm{f}}\left(g_{0}\right)\langle x\rangle_{\mathrm{f}}^{\text {bare }},\langle x\rangle_{\mathrm{g}}=Z_{\mathrm{g}}\left(g_{0}\right)\langle x\rangle_{\mathrm{g}}^{\text {bare }} .
$$

The particular discretization of $\bar{T}_{00}^{\mathrm{g}}$ appearing in the lattice sum rule is the bare plaquette one. The normalization factor $Z_{\mathrm{g}}\left(g_{0}\right)$ and its fermionic analog $Z_{\mathrm{f}}\left(g_{0}\right)$ are given by the so-called anisotropy coefficients used in thermodynamic studies. The MSR thus holds even in an 'unrenormalized form' where $\langle x\rangle_{\mathrm{f}, \mathrm{g}}$ do not separately have a continuum limit; their renormalization is performed in the next section.

\section{Renormalization of $\langle x\rangle_{\mathrm{g}}$}

Recall that, in QCD, the renormalization pattern in the singlet sector reads [12]

$$
\left[\begin{array}{l}
\bar{T}_{00}^{\mathrm{g}}(\mu) \\
\bar{T}_{00}^{\mathrm{f}}(\mu)
\end{array}\right]=\left[\begin{array}{lr}
Z_{\mathrm{gg}} & 1-Z_{\mathrm{ff}} \\
1-Z_{\mathrm{gg}} & Z_{\mathrm{ff}}
\end{array}\right]\left[\begin{array}{l}
\bar{T}_{00}^{\mathrm{g}}\left(g_{0}\right) \\
\bar{T}_{00}^{\mathrm{f}}\left(g_{0}\right)
\end{array}\right],
$$


provided $\bar{T}_{00}^{\mathrm{f,g}}\left(g_{0}\right)$ are normalized so that Eqs. (1.7 1.9) hold. In lattice regularization, this requires the scheme-independent $Z_{\mathrm{g}}\left(g_{0}\right)$ and $Z_{\mathrm{f}}\left(g_{0}\right)$ factors, whereas $Z_{\mathrm{gg}}$ and $Z_{\mathrm{ff}}$ are scheme-dependent functions of $\left(a \mu, g_{0}\right)$. The renormalization group equation then takes the form

$$
\mu \partial_{\mu}\left[\begin{array}{l}
\langle x\rangle_{\mathrm{g}}\left(\mu^{2}\right) \\
\langle x\rangle_{\mathrm{f}}\left(\mu^{2}\right)
\end{array}\right]=-\bar{g}^{2}(\mu)\left[\begin{array}{lr}
c_{\mathrm{gg}}(\bar{g}) & -c_{\mathrm{ff}}(\bar{g}) \\
-c_{\mathrm{gg}}(\bar{g}) & c_{\mathrm{ff}}(\bar{g})
\end{array}\right]\left[\begin{array}{l}
\langle x\rangle_{\mathrm{g}}\left(\mu^{2}\right) \\
\langle x\rangle_{\mathrm{f}}\left(\mu^{2}\right)
\end{array}\right]
$$

with $\mu \partial_{\mu} \log \left[Z_{\mathrm{gg}}+Z_{\mathrm{ff}}-1\right]=-\bar{g}^{2}\left[c_{\mathrm{gg}}+c_{\mathrm{ff}}\right]$ and $c_{\mathrm{gg}, \mathrm{ff}}(\bar{g}=0)=\frac{N_{\mathrm{f}}}{12 \pi^{2}}$, $\frac{4}{9 \pi^{2}}$ respectively [13]. Besides the zero-mode $\bar{T}_{00}$, the linear combination $[1+\tau(\mu)] \bar{T}_{00}^{\mathrm{g}}(\mu)+\tau(\mu) \bar{T}_{00}^{\mathrm{f}}(\mu)$ renormalizes multiplicatively with anomalous dimension $-\bar{g}^{2}\left[c_{\mathrm{ff}}+c_{\mathrm{gg}}\right]$, where $\mu \partial_{\mu} \tau=-\bar{g}^{2}\left[\left(c_{\mathrm{ff}}+c_{\mathrm{gg}}\right) \tau+c_{\mathrm{ff}}\right]$. Note that the asymptotic glue momentum fraction is given by $c_{\mathrm{ff}}(0) /\left[c_{\mathrm{ff}}(0)+c_{\mathrm{gg}}(0)\right]=Z_{\mathrm{gg}}(\infty)=$ $1-Z_{\mathrm{ff}}(\infty)=-\tau(\infty)=16 /\left[16+3 N_{\mathrm{f}}\right]$

In the quenched approximation, $Z_{\mathrm{gg}}=1$ due to the absence of quark loops [17, 16]. This implies that the singlet quark operator renormalizes multiplicatively and with the same $Z$-factor, computed non-perturbatively in [14], as the non-singlet operator:

$$
\begin{aligned}
\langle x\rangle_{\mathrm{g}}\left(\mu^{2}\right) & =\langle x\rangle_{\mathrm{g}}+\left[1-Z_{\mathrm{ff}}\left(a \mu, g_{0}\right)\right]\langle x\rangle_{\mathrm{f}} \\
\langle x\rangle_{\mathrm{f}}\left(\mu^{2}\right) & =Z_{\mathrm{ff}}\left(a \mu, g_{0}\right)\langle x\rangle_{\mathrm{f}} .
\end{aligned}
$$

Disregarding disconnected diagrams, $\langle x\rangle_{\mathrm{f}}^{\text {bare }}$ has been computed in [15] at the bare parameters $(\beta=6, \kappa=0.1530)$. The product $Z_{\mathrm{ff}}\left(a \mu, g_{0}\right) Z_{\mathrm{f}}\left(g_{0}\right)=0.99(4)$ (in the $\overline{M S}$-scheme at $\mu=2 \mathrm{GeV}$ ) is known from [14, 15]. The factor $Z_{\mathrm{f}}\left(g_{0}\right)=1+\mathrm{O}\left(g_{0}^{2}\right)$ is as yet unknown beyond tree level. If we allow for a conservative error, based on the typical size of one-loop corrections, $Z_{\mathrm{f}}\left(g_{0}\right)=1.0(2)$, our final result is

$$
\langle x\rangle_{\mathrm{g}}^{(\pi)}\left(\mu_{\overline{M S}}^{2}=4 \mathrm{GeV}^{2}\right)=0.37(8)(12) \quad\left(M_{\pi}=890 \mathrm{MeV}\right),
$$

where the first error is statistical and the second comes from the uncertainty in $Z_{\mathrm{f}}\left(g_{0}\right)$. We observe no significant quark-mass dependence, and given the uncertainties, we do not attempt a chiral extrapolation.

Our result for the glue momentum fraction in a (heavy) pion is compatible with phenomenological determinations [18, 19], $\langle x\rangle_{g}^{\overline{M S}}=0.38(5)$ at $Q^{2}=4 \mathrm{GeV}^{2}$, based on Drell-Yan, prompt photoproduction, and the model assumption that sea quarks carry $10-20 \%$ of the momentum. The agreement suggests a mild quark-mass dependence, but only a calculation in full QCD and at smaller masses can substantiate this. Our result at $Q^{2}=4 \mathrm{GeV}^{2}$ lies clearly below the $N_{\mathrm{f}}=3$ asymptotic glue momentum fraction of 0.64 . The fact that our result and the valence quark momentum fraction, computed in [15], add up to $0.99(8)(12)$ suggests that the omitted disconnected diagrams are small.

\section{Conclusion}

We have computed the glue momentum fraction $\langle x\rangle_{\mathrm{g}}$ in a pion of mass $0.6 \mathrm{GeV}<M_{\pi}<$ $1.06 \mathrm{GeV}$ using quenched lattice QCD simulations. We find $37(8)(12) \%$ at $\mu_{\overline{M S}}=2 \mathrm{GeV}$, a result compatible with phenomenological determinations [18, 19]. 
Although it appears difficult to achieve precision at the percent level, the present method is applicable to full QCD with dynamical quarks. Presently the larger uncertainty comes from the normalization of the quark contribution to the renormalized $\langle x\rangle_{\mathrm{g}}$, and could be reduced significantly by a one-loop calculation.

We also evaluated the bare trace anomaly contribution to the pion's mass in the same framework [3]. The counterterm remains to be calculated, but it will ultimately be preferable to use chiral fermions to avoid mixing with the lower dimensional fermion operator.

Finally, we remark that the freedom of choosing a numerically advantageous discretization of $\bar{T}_{\mu \nu}$ has not been fully exploited in previous lattice simulations. The improvement that was essential in the present computation of the pion momentum fraction can be carried over to fully dynamical calculations and the exploration of other observables, such as the gluon contribution to the nucleon spin. It is also particularly promising for thermodynamic studies of pressure, energy density and transport coefficients.

Acknowledgments. This work was supported in part by funds provided by the U.S. Department of Energy under cooperative research agreement DE-FG02-94ER40818.

\section{References}

[1] X. D. Ji, Phys. Rev. Lett. 74, 1071 (1995) [arXiv:hep-ph/9410274].

[2] M. Göckeler et al., Nucl. Phys. Proc. Suppl. 53, 324 (1997) [arXiv:hep-lat/9608017].

[3] H. B. Meyer and J. W. Negele, arXiv:0707.3225 [hep-lat].

[4] K.G. Wilson, Phys. Rev. D 10, 2445 (1974).

[5] S. Necco and R. Sommer, Nucl. Phys. B 622 (2002) 328.

[6] Y. Chen et al., Phys. Rev. D 73, 014516 (2006) [arXiv:hep-lat/0510074].

[7] B. Lucini, M. Teper and U. Wenger, JHEP 0401, 061 (2004).

[8] M. Lüscher, S. Sint, R. Sommer and P. Weisz, Nucl. Phys. B 478, 365 (1996) [arXiv:hep-lat/9605038].

[9] A. Hasenfratz and F. Knechtli, Phys. Rev. D 64, 034504 (2001) [arXiv:hep-lat/0103029].

[10] H. B. Meyer, arXiv:0704.1801 [hep-lat].

[11] H. B. Meyer, Nucl. Phys. B 760, 104 (2007) [arXiv:hep-lat/0609007].

[12] X. D. Ji, Phys. Rev. D 52, 271 (1995) [arXiv:hep-ph/9502213].

[13] D. J. Gross and F. Wilczek, Phys. Rev. D 9, 980 (1974); H. Georgi and H. D. Politzer, Phys. Rev. D 9 , 416 (1974).

[14] M. Guagnelli, K. Jansen, F. Palombi, R. Petronzio, A. Shindler and I. Wetzorke [Zeuthen-Rome / ZeRo Collaboration], Nucl. Phys. B 664, 276 (2003) [arXiv:hep-lat/0303012].

[15] M. Guagnelli, K. Jansen, F. Palombi, R. Petronzio, A. Shindler and I. Wetzorke [Zeuthen-Rome (ZeRo) Collaboration], Eur. Phys. J. C 40, 69 (2005) [arXiv:hep-lat/0405027].

[16] F. Palombi, R. Petronzio and A. Shindler, Nucl. Phys. B 637, 243 (2002) [arXiv:hep-lat/0203002].

[17] S. Capitani and G. Rossi, Nucl. Phys. B 433, 351 (1995) [arXiv:hep-lat/9401014].

[18] P. J. Sutton, A. D. Martin, R. G. Roberts and W. J. Stirling, Phys. Rev. D 45, 2349 (1992).

[19] M. Glück, E. Reya and I. Schienbein, Eur. Phys. J. C 10, 313 (1999) [arXiv:hep-ph/9903288]. 